\title{
Characteristics of Asians in the FOURIER Trial Using Evolocumab
}

\author{
Masaaki Miyata, MD, PhD
}

$\mathbf{T}$ The first epoch-making discovery in lipid-lowering medicine for hypercholesterolemia was identification of 3-hydroxy-3-methylglutaryl coenzyme A (HMG-CoA) reductase (i.e., statin) by Dr. Akira Endo, and the second one was the proprotein convertase subtilisin kexin 9 (PCSK9) antibody inhibitor. PCSK9 is a member of the subtilisin serine protease family and plays a role in the degradation of the low-density lipoprotein (LDL) receptor. ${ }^{1}$ Loss-of-function variants in the PCSK9 gene are associated with the reduction in LDL-cholesterol and overall coronary artery disease (CAD). ${ }^{2}$ The Further Cardiovascular Outcomes Research with PCSK9 Inhibition in Subjects with Elevated Risk (FOURIER) trial was a randomized, double-blind, placebo-controlled trial involving 27,564 patients with atherosclerotic cardiovascular disease and LDL-cholesterol levels of $\geq 70 \mathrm{mg} / \mathrm{dL}$ who were receiving

\section{Article p????}

statin therapy. It demonstrated that inhibition of PCSK9 with evolocumab, the monoclonal antibody for PCSK9, decreased LDL cholesterol levels from $92 \mathrm{mg} / \mathrm{dL}$ to $30 \mathrm{mg} / \mathrm{dL}$ and reduced the risk of cardiovascular events. ${ }^{3}$

Because of differences in genetics, physical size, drug metabolism, usage of high-intensity statins, and incidence of CAD between Asians and other races, it is important to compare the effects and adverse events of evolocumab between Asians and others. In this issue of the Journal, Keech et al evaluate the efficacy and safety of evolocumab among Asians vs. other subjects using data from the FOURIER trial, and conclude that the use of evolocumab robustly lowers LDL-cholesterol and is equally safe and efficacious

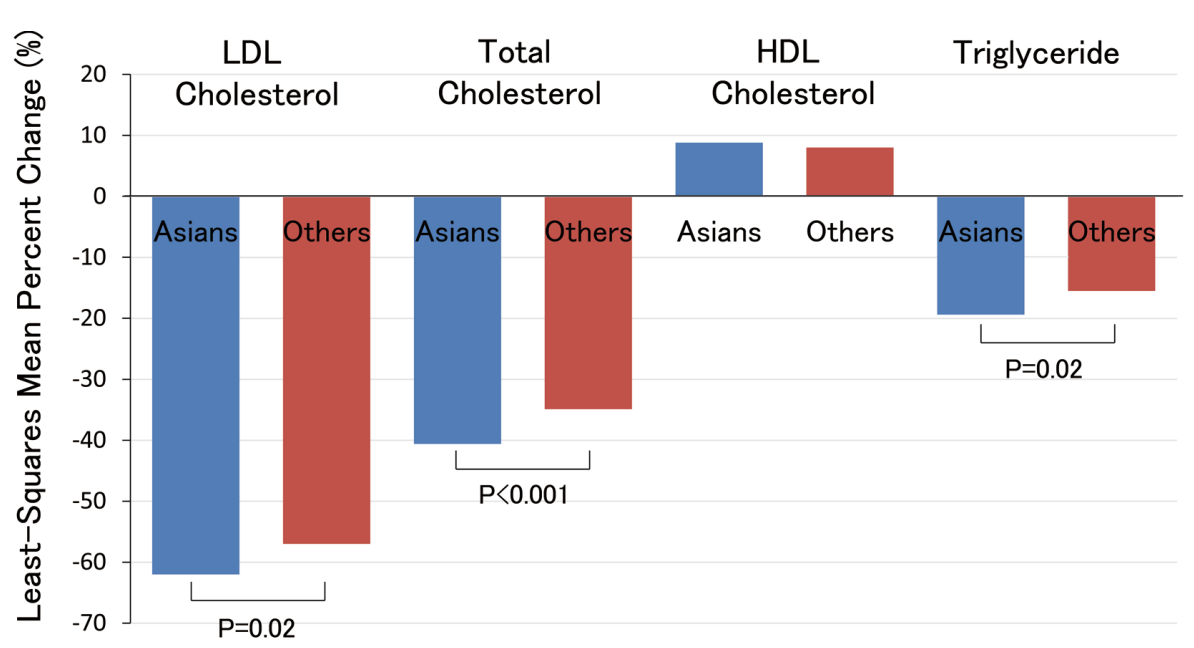

Figure. Comparison of least-squares mean percentage change in lipid by Asians vs. others. Least-squares mean (adjusted mean) is based on a repeated measures ANCOVA model including treatment, randomization strata ( $\mathrm{LDL} \geq 85 \mathrm{mg} / \mathrm{dL}$ and region), visit and the interaction of treatment-by-visit interaction with the baseline value. Least-squares mean percent change shows the treatment difference between evolocumab and placebo groups. HDL, high-density lipoprotein; LDL, low-density lipoprotein. (Figure created using the data of Keech et al ${ }^{4}$ with permission.)

The opinions expressed in this article are not necessarily those of the editors or of the Japanese Circulation Society. Received March 21, 2021; accepted March 22, 2021; J-STAGE Advance Publication released online May 13, 2021

School of Health Sciences, Faculty of Medicine, Kagoshima University, Kagoshima, Japan

Mailing address: Masaaki Miyata, MD, PhD, School of Health Sciences, Faculty of Medicine, Kagoshima University, 8-35-1

Sakuragaoka, Kagoshima 890-8544, Japan. E-mail: miyatam@m3.kufm.kagoshima-u.ac.jp

All rights are reserved to the Japanese Circulation Society. For permissions, please e-mail: cj@j-circ.or.jp

ISSN-1346-9843 
in lowering the risk of cardiovascular events in Asians as it is in others. ${ }^{4}$

Here, I would like to highlight several concerns about this second analysis of the FOURIER trial between Asian and other subjects. The lowering effect of evolocumab on LDL-cholesterol, total cholesterol, and triglycerides was significantly larger in Asians compared with other races (Figure). However, there were no significant differences between the 2 groups in the adverse effects of evolocumab, such as rhabdomyolysis, hemorrhagic stroke, new onset diabetes mellitus, or cognitive decline. Although the incidence of CAD in Asians is lower than in others, the reductions in the primary endpoints, including major adverse cardiovascular events, defined as the composite of cardiovascular death, myocardial infarction, stroke, hospitalization for unstable angina, or coronary revascularization, and the key secondary endpoints, such as the composite of cardiovascular death, myocardial infarction, or stroke, were similar between Asians and other subjects. ${ }^{4}$

As shown in a previous subanalysis of FOURIER focusing on the efficacy and safety of achieving very low LDLcholesterol concentrations, there was a monotonic relationship between achieved LDL-cholesterol and major cardiovascular outcomes down to LDL-cholesterol concentrations of $<7.7 \mathrm{mg} / \mathrm{dL}$. Conversely, there were no safety concerns with very low LDL-cholesterol concentrations over a median of 2.2 years. These results support further LDL-cholesterol lowering in patients with cardiovascular disease to well below current recommendations. ${ }^{5}$

Another subanalysis of FOURIER demonstrated that evolocumab was equally effective in reducing cardiovascular events in patients with stable atherosclerotic cardiovascular disease, regardless of whether the baseline LDL-C was $<70$ or $\geq 70 \mathrm{mg} / \mathrm{dL}$ and whether the background statin was of maximal or submaximal potency. ${ }^{6}$ Keech et al show that the use of high-intensity statins and the baseline LDLcholesterol levels were significantly lower in Asians compared with others. The mechanism of the stronger lipid-lowering effect of evolocumab in Asians compared with others has not been elucidated. Therefore, further studies are needed to clarify this mechanism, including PCSK9 activity, genetics, body weight, and drug metabolism.

A genetic subanalysis of FOURIER using a 27-singlenucleotide polymorphism genetic risk score ${ }^{7}$ reported that patients without multiple clinical risk factors or high genetic risk had a low event rate and did not appear to derive benefit from evolocumab over 2.3 years. Conversely, patients with multiple clinical risk factors but without high genetic risk had intermediate risk and intermediate risk reduction. Patients with high genetic risk, regardless of clinical risk, had a high event rate and derived the greatest relative and absolute benefit from evolocumab, which mitigated this risk. ${ }^{7}$ Therefore, precision medicine using genetic analysis may be useful to decide the indication of evolocumab in the near future. Moreover, these genetic analyses could be able to clarify the genetic differences among races.

\section{Conflicts of Interests}

The author declares no conflicts of interest.

\section{References}

1. Maxwell KN, Fisher EA, Breslow JL. Overexpression of PCSK9 accelerates the degradation of the LDLR in a post-endoplasmic reticulum compartment. Proc Natl Acad Sci USA 2005; 102: 2069-2074.

2. Cohen JC, Boerwinkle E, Mosley TH, Hobbs HH. Sequence variations in PCSK9, low LDL, and protection against coronary heart disease. N Engl J Med 2006; 354: 1264-1272.

3. Sabatine MS, Giugliano RP, Keech AC, Honarpour N, Wiviott $\mathrm{SD}$, Murphy SA, et al. Evolocumab and clinical outcomes in patients with cardiovascular disease. $N$ Engl J Med 2017; 376: $1713-1722$

4. Keech AC, Oyama K, Sever PS, Tang M, Murphy SA, Hirayama A, et al; on behalf of the FOURIER Study Group. Efficacy and safety of long-term evolocumab use among Asian subjects: A subgroup analysis of the further cardiovascular outcomes research with PCSK9 inhibition in subjects with elevated risk (FOURIER) trial. Circ J, doi:10.1253/circj.CJ-20-1051.

5. Giugliano RP, Pedersen TR, Park JG, De Ferrari GM, Gaciong ZA, Ceska R, et al. Clinical efficacy and safety of achieving very low LDL-cholesterol concentrations with the PCSK9 inhibitor evolocumab: A prespecified secondary analysis of the FOURIER trial. Lancet 2017; 390: 1962-1971.

6. Giugliano RP, Keech A, Murphy SA, Huber K, Tokgozoglu SL, Lewis BS, et al. Clinical efficacy and safety of evolocumab in high-risk patients receiving a statin: Secondary analysis of patients with low LDL cholesterol levels and in those already receiving a maximal-potency statin in a randomized clinical trial. JAMA Cardiol 2017; 2: 1385-1391.

7. Marston NA, Kamanu FK, Nordio F, Gurmu Y, Roselli C, Sever PS, et al. Predicting benefit from evolocumab therapy in patients with atherosclerotic disease using a genetic risk score. Circulation 2020; 141: 616-623. 